\title{
Statistical Models and Methods for Dependence in
}

\section{Insurance Data}

\author{
Stephan Haug*, Claudia Klüppelberg ${ }^{\dagger}$ and Liang Peng ${ }^{\ddagger}$
}

\begin{abstract}
Copulas are becoming a quite flexible tool in modeling dependence among the components of a multivariate vector. In order to predict extreme losses in insurance and finance, extreme value copulas and tail copulas play a more important role than copulas. In this paper, we review some estimation and testing procedures for both, extreme value copulas and tail copulas, which received much less attention in the literature than corresponding studies of copulas.
\end{abstract}

Keywords and phrases: Copula, dependence modelling, extreme dependence, extreme risk, extreme value copula, inference for copulas, interval estimation, multivariate statistics, point estimation, risk estimation, risk modeling, tail copula, tail dependence coefficient, hypothesis testing.

NOTICE: This is the author's version of a work that was accepted for publication in Journal of the Korean Statistical Society. Changes resulting from the publishing process, such as peer review, editing, corrections, structural formatting, and other quality control mechanisms may not be reflected in this document. Changes may have been made to this work since it was submitted for publication. A definitive version was subsequently published in Volume 40, Issue 2, pages 125-139.

\section{Introduction}

Dependence among variables is a relevant feature, when analyzing financial losses and insurance claims data. Fitting a parametric family to a univariate variable is much easier than to a multivariate vector. By separating from marginals, copulas have become a useful tool in modeling dependence in particular for risk management. Some recent review papers on copulas and their extensions include Kolve, Anjos and Mendes [37], Mikosch [40], Embrechts [15] and Patton [44]. Although there exists a huge amount of studies on constructing various copulas and statistical inference for copulas, the study of multivariate

\footnotetext{
*Zentrum Mathematik, Technische Universität München, Boltzmannstraße 3, D-85748 Garching, Germany.

Email: $\{$ haug\}@ma.tum.de

${ }^{\dagger}$ Zentrum Mathematik and Institute for Advanced Study, Technische Universität München, Boltzmannstrasse 3, 85748

Garching, Germany

${ }^{\ddagger}$ School of Mathematics, Georgia Institute of Technology, Atlanta, GA 30332-0160, USA
} 
extremes related with copulas is quite behind in comparison with the research on copulas. After a quick summary on estimation and hypothesis testing procedures for copulas, we review statistical inference for extreme value copulas and tail copulas, which play important roles in predicting extreme events.

The paper aims at statisticians in academia and industry, who are involved in risk assessment, dealing in particular with dependent risks. We present statistical methods relevant for multivariate risk estimation, give ample references for further reading, and present the methods at a specific data example. The statistical analysis is performed with the statistical software package $\mathrm{R}$, which is an open source software; for details see below.

Let $\left(X_{1}, \ldots, X_{d}\right)^{\top}$ be a random vector with distribution function $F$, and $F_{j}$ denote the marginal distribution function of $X_{j}$ for $j=1, \ldots, d$. Throughout the paper we will assume that the marginal distributions are continuous. In that case the copula of $\left(X_{1}, \ldots, X_{d}\right)^{\top}$ is uniquely defined as

$$
C_{F}\left(u_{1}, \ldots, u_{d}\right)=P\left(F_{1}\left(X_{1}\right) \leq u_{1}, \ldots, F_{d}\left(X_{d}\right) \leq u_{d}\right)
$$

for $\left(u_{1}, \ldots, u_{d}\right)^{\top} \in[0,1]^{d}$. How to infer a copula plays an important role in various applications. More detailed review on point estimation, interval estimation and goodness-of-fit tests is given in Section 2 .

In insurance and finance, modeling extreme events is of high importance since, although they happen rarely, if they happen, then they often infer huge losses for industry and society. An extreme event is characterized by the fact that it is more disastrous than any previously observed event. Extreme value theory deals with this problem by extrapolating from past observations into a far tail region. This obviously sets limits to purely nonparametric copula estimation. On the other hand, if a parametric copula is employed, extrapolation will become quite sensitive. Hence, it is desirable to have some "copulas", which have an intrinsic extrapolation property so that nonparametric estimation can be applied. Based on multivariate extreme value theory, both extreme value copulas and tail copulas can be used to predicting extreme events.

To define an extreme value copula we consider the vector of componentwise maxima

$$
\mathbf{M}_{n}:=\left(M_{n 1}, \ldots, M_{n d}\right)^{\top}
$$

of a given sample $\mathbf{X}_{i}=\left(X_{i 1}, \ldots, X_{i d}\right)^{\top}$ for $i=1, \ldots, n$, of i.i.d. random vectors with common distribution function $F$. It follows that the copula $C_{\mathbf{M}_{n}}$ of $\mathbf{M}_{n}$ is given by

$$
C_{\mathbf{M}_{n}}\left(u_{1}, \ldots, u_{d}\right)=C_{F}\left(u_{1}^{1 / n}, \ldots, u_{d}^{1 / n}\right)^{n}
$$

for $\left(u_{1}, \ldots, u_{d}\right)^{\top} \in[0,1]^{d}$. 
A copula $C$ is now called an extreme value copula, if there exists a copula $C_{F}$ such that for all $\left(u_{1}, \ldots, u_{d}\right)^{\top} \in[0,1]^{d}$,

$$
\lim _{n \rightarrow \infty} C_{F}\left(u_{1}^{1 / n}, \ldots, u_{d}^{1 / n}\right)^{n}=C\left(u_{1}, \ldots, u_{d}\right)
$$

The class of extreme value copulas coincides with the class of max-stable copulas; i.e., a copula $C$ is an extreme value copula if and only if for all $n \in \mathbb{N}$

$$
C\left(u_{1}, \ldots, u_{d}\right)=C\left(u_{1}^{1 / n}, \ldots, u_{d}^{1 / n}\right)^{n}
$$

for $\left(u_{1}, \ldots, u_{d}\right)^{\top} \in[0,1]^{d}$; see e.g., Gudendorf and Segers [27].

Due to this special property of the extreme value copula, one can extrapolate data into a far tail region in order to estimate the probability of an extreme event. We will review some estimation and testing procedures for an extreme value copula in Section 3.

When the extreme event is really far away from the data range, condition (1) may be relaxed to be true in an asymptotic way. This gives the so-called upper tail copula or upper tail dependence function defined as

$$
T\left(x_{1}, \ldots, x_{d}\right)=\lim _{t \rightarrow 0} t^{-1} P\left(F_{1}\left(X_{1}\right) \geq 1-x_{1} t, \ldots, F_{d}\left(X_{d}\right) \geq 1-x_{d} t\right)
$$

for $\left(x_{1}, \ldots, x_{d}\right)^{\top} \in(0, \infty)^{d}$, if the limit exists. Similarly, a lower tail copula or lower tail dependence function and other parts of tail copulas can be defined, see Joe, Li and Nikoloulopoulos [33]. Obviously, a tail copula is not a copula. Since we are interested in large losses, we concentrate in this paper on the upper tail copula, which we call simply tail copula from now on. Since a tail copulas is defined as a limit, the prediction of an extreme event based on the tail copula becomes more robust than using the copula or extreme value copula, when the extreme event is far away from the data range. More details are given in Section 4.

We organize this review paper as follows. Right at the end of this introduction we present our data and some summary statistics. In Section 2 we explain our concepts for copulas in general, before we move on to the more interesting objects for risk management; i.e., extreme value copulas and tail copulas. Section 3 presents estimation and testing procedures for an extreme value copula. Review on inference for tail copulas is given in Section 4. We conclude with an outlook in Section 5.

To illustrate these methods empirically we will analyze the Danish fire insurance claims at the end of each section with the methods just presented. This data set is available at www.ma.hw.ac.uk/ mcneil/. As described there, the data were collected at the Copenhagen Reinsurance Company and comprise 2167 fire losses over the period 1980 to 1990. They have been adjusted for inflation to reflect 1985 
values and are expressed in millions of Danish Kroner. This data set has first been considered by Rytgaard [55], see also Embrecht, Klüppelberg and Mikosch [16, Example 6.2.9 and further]. The data is three-dimensional consisting of loss to building, loss to contents and loss to profits. Since the last variable has rarely non-zero value, we restrict our analyses to the first two variables. The claims are recorded only if the sum of losses to building, contents and profits is greater or equal to 1 million Danish Kroner (DK). This introduces a negative dependence between the components. Because of that we will only consider those observations with both components being greater than or equal to 1 million DK. This implies that we are not working with the copula $P\left(F_{1}\left(X_{1}\right) \leq u_{1}, F_{2}\left(X_{2}\right) \leq u_{2}\right)$, $\left(u_{1}, u_{2}\right)^{\top} \in[0,1]^{2}$, but with the conditional copula

$$
P\left(F_{1 \mid \geq 1}\left(X_{1}\right) \leq u_{1}, F_{2 \mid \geq 1}\left(X_{2}\right) \leq u_{2}\right), \quad\left(u_{1}, u_{2}\right)^{\top} \in[0,1]^{2},
$$

where

$$
F_{1 \mid \geq 1}\left(x_{1}\right):=P\left(X_{1} \leq x_{1} \mid X_{1} \geq 1\right) \quad \text { and } \quad F_{2 \mid \geq 1}\left(x_{2}\right):=P\left(X_{2} \leq x_{2} \mid X_{2} \geq 1\right)
$$

with $X_{1}=$ loss to building and $X_{2}=$ loss to contents. For ease of notation we will write in the following $F_{1}$ and $F_{2}$ for the conditional distribution functions $F_{1 \mid \geq 1}$ and $F_{2 \mid \geq 1}$, respectively. Due to this data selection our reduced sample size consists of $n=301$ observations. Figure 1 shows a scatterplots of the resulting data set.

An analysis of the data in their dynamic context: Esmaeili and Klüppelberg [18] fitted a bivariate compound Poisson process to the data by maximum likelihood estimation; a Bayesian analysis has been performed in Gebhard, Müller and Böcker [20].

The data set will be analyzed using the statistical software package R ( R Development Core Team 2010). For parametric estimation and goodness-of-fit test we will use the copula package, and the empirical likelihood ratios for the interval estimation are computed with the emplik package. Both are available from the Comprehensive R Archive Network at http://cran.R-project.org. The specific $\mathrm{R}$ code to compute the confidence intervals can be obtained upon request from the third author. By using $\mathrm{R}$ we obtain the following summary statistics.

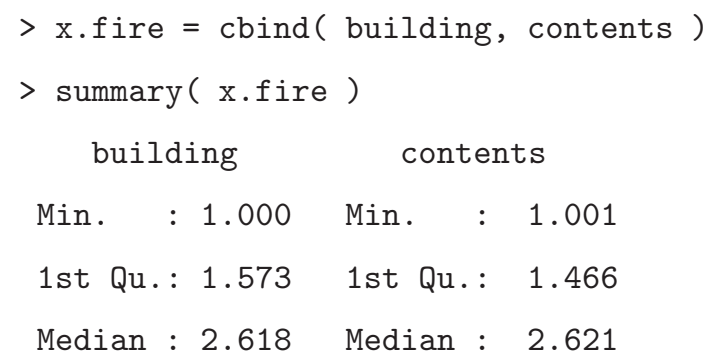



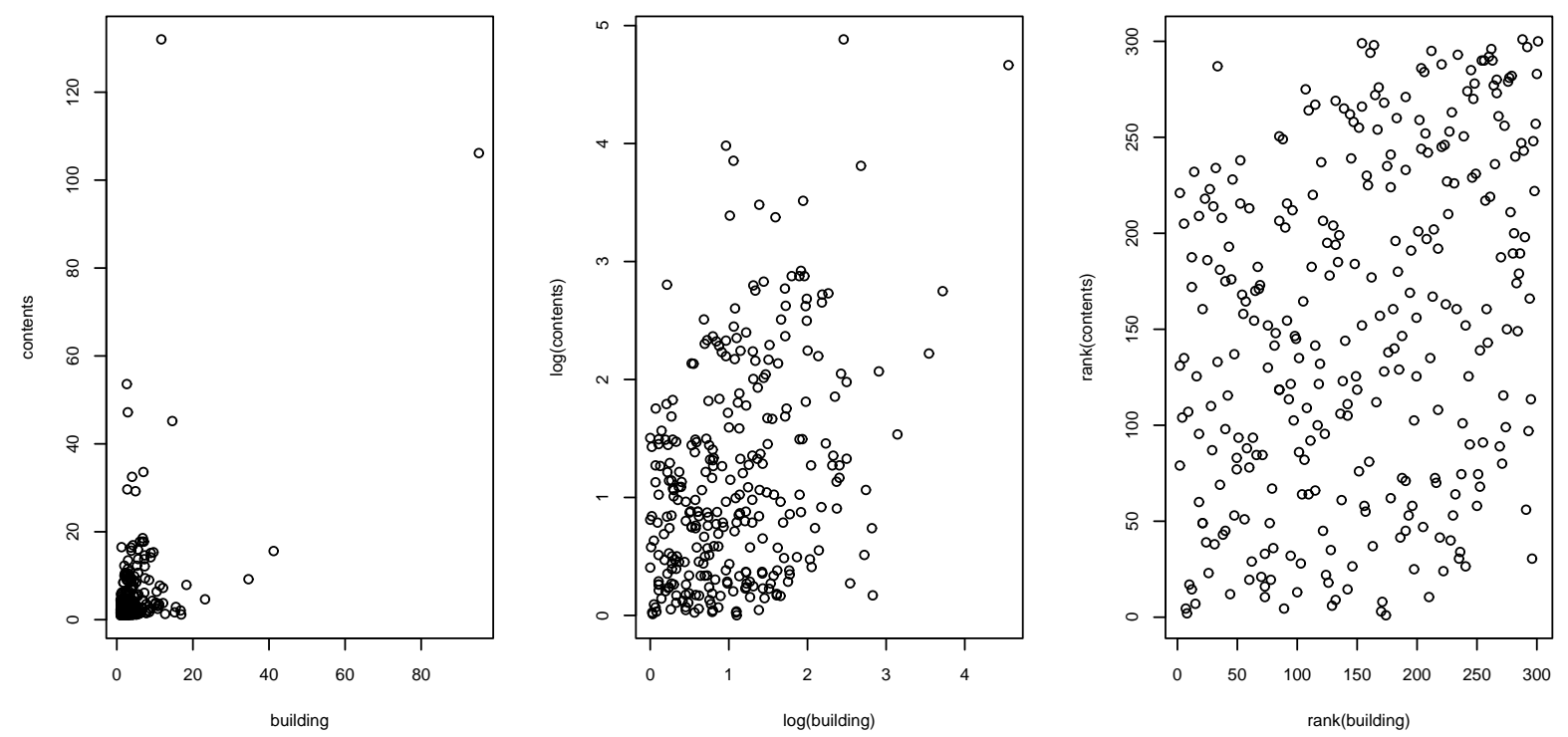

FiguRE 1. Scatterplot of the Danish fire insurance data (left), of the log transformed data (middle) and of the ranks of the data (right).

$\begin{array}{llll}\text { Mean }: 4.215 & \text { Mean } & 5.656 \\ \text { 3rd Qu.: } 4.452 & \text { 3rd Qu.: } 5.289 \\ \text { Max. } & : 95.168 & \text { Max. } & \text { : } 132.013\end{array}$

Estimators for the Pearson correlation and Kendall's rank correlation are the following.

$>c(\operatorname{cor}(x . f i r e)[1,2], \operatorname{cor}(x . f i r e$, method="kendall") $[1,2])$

[1] 0.51244620 .2110319

Since linear correlation is not a suitable dependence measure in this context (see e.g. Embrechts, McNeil and Straumann [17]), we concentrate on Kendall's tau and test if it is greater than zero.

$>$ cor.test ( building, contents, method = "kendall", alternative = "greater" )

Kendall's rank correlation tau

data: building and contents

$z=5.4516, p$-value $=2.495 e-08$

alternative hypothesis: true tau is greater than 0

sample estimate:

$\operatorname{tau}=0.2110319$ 
Figure 1 already suggested some positive dependence between the loss to building and the loss to contents. This is confirmed by an estimated Kendall's tau, which is significantly greater than zero.

\section{Inference for copulas}

\subsection{Point estimation}

For estimating the copula of a random sample $\left(\mathbf{X}_{1}, \ldots, \mathbf{X}_{n}\right)^{\top}$ from the $d$-variate distribution function $F$ with copula $C$, both nonparametric and parametric estimation have been studied in the literature. The simplest nonparametric estimator of a copula $C$ at $\left(u_{1}, \ldots, u_{d}\right)^{\top} \in[0,1]^{d}$ is the empirical copula

$$
\mathbb{C}_{n}\left(u_{1}, \ldots, u_{d}\right)=\frac{1}{n} \sum_{i=1}^{n} \mathbb{1}\left(U_{i 1} \leq u_{1}, \ldots, U_{i d} \leq u_{d}\right),
$$

where $U_{i j}:=\frac{n}{n+1} \mathbb{F}_{n j}\left(X_{i j}\right)$ for $i=1, \ldots, n$ and $j=1, \ldots, d$ are the so-called pseudo observations, $\mathbb{F}_{n j}(x)=\frac{1}{n} \sum_{i=1}^{n} \mathbb{1}\left(X_{i j} \leq x\right)$ for $j=1, \ldots, d$, are the marginal empirical distribution functions and $\mathbb{1}(\cdot)$ denotes the indicator function. A smoothed version of $\mathbb{C}_{n}$ can be defined as

$$
\hat{\mathbb{C}}_{n}\left(u_{1}, \ldots, u_{d}\right)=\frac{1}{n} \sum_{i=1}^{n} K_{d}\left(\frac{u_{1}-\mathbb{F}_{n 1}\left(X_{i 1}\right)}{h_{1}}, \ldots, \frac{u_{d}-\mathbb{F}_{n d}\left(X_{i d}\right)}{h_{d}}\right),
$$

where $K_{d}\left(x_{1}, \ldots, x_{d}\right):=\int_{-\infty}^{x_{1}} \ldots \int_{-\infty}^{x_{d}} k_{d}\left(y_{1}, \ldots, y_{d}\right) d y_{d} \ldots d y_{1}$ and $k_{d}: \mathbb{R}^{d} \rightarrow \mathbb{R}$ is a kernel function with $\int k_{d}(\mathbf{y}) d \mathbf{y}=1$ and $h_{j}=h_{j}(n)>0$ is a bandwidth for $j=1, \ldots, d$. We refer to Fremanian, Radulović and Wegkamp [19] and Omelka, Gijbels and Veraverbeke [42] for a study of asymptotic properties of the empirical copula and smoothed copula estimation.

For parametric estimation, we focus on fitting a parametric family to the empirical copula, leaving the marginals free. We recall some popular bivariate parametric copula families. In particular, we give the definition of the Gumbel, Clayton, Frank, normal and Plackett copula for $\left(u_{1}, u_{2}\right)^{\top} \in[0,1]^{2}$.

- Gumbel copula: for $\theta \in[1, \infty)$,

$$
C^{G u}\left(u_{1}, u_{2} ; \theta\right)=\exp \left(-\left(\left(-\log \left(u_{1}\right)\right)^{\theta}+\left(-\log \left(u_{2}\right)\right)^{\theta}\right)^{1 / \theta}\right) .
$$

- Clayton copula: for $\theta \in(0, \infty)$,

$$
C^{C l}\left(u_{1}, u_{2} ; \theta\right)=\left(u_{1}^{-\theta}+u_{2}^{-\theta}-1\right)^{-1 / \theta} .
$$

- Frank copula: for $\theta \in \mathbb{R} \backslash\{0\}$,

$$
C^{F r}\left(u_{1}, u_{2} ; \theta\right)=-\frac{1}{\theta} \log \left(1+\frac{\left(\exp \left(-\theta u_{1}\right)-1\right)\left(\exp \left(-\theta u_{2}\right)-1\right)}{\exp (-\theta)-1}\right) .
$$


- Normal copula: for $\theta \in(-1,1)$,

$$
C^{N o}\left(u_{1}, u_{2} ; \theta\right)=\frac{1}{2 \pi \sqrt{1-\theta^{2}}} \int_{-\infty}^{\Phi^{-1}\left(u_{1}\right)} \int_{-\infty}^{\Phi^{-1}\left(u_{2}\right)} \exp \left(\frac{-\left(x_{1}^{2}-2 \theta x_{1} x_{2}+x_{2}^{2}\right)}{2\left(1-\theta^{2}\right)}\right) d x_{1} d x_{2}
$$

where $\Phi$ denotes the distribution function of the standard normal distribution with inverse $\Phi^{-1}$.

- Plackett copula: for $\theta \in \mathbb{R}_{+} \backslash\{1\}$,

$$
C^{P l}\left(u_{1}, u_{2} ; \theta\right)=\frac{\left(1+(\theta-1)\left(u_{1}+u_{2}\right)\right)-\sqrt{\left(1+(\theta-1)\left(u_{1}+u_{2}\right)\right)^{2}-4 u_{1} u_{2} \theta(\theta-1)}}{2(\theta-1)},
$$

and $C^{P l}\left(u_{1}, u_{2} ; 1\right)=u_{1} u_{2}$.

Specifically, we assume that the copula $C$ comes from the parametric family $C(\cdot ; \boldsymbol{\theta})$, and is absolutely continuous with density $c(\cdot ; \boldsymbol{\theta})$, where $\boldsymbol{\theta} \in \mathbf{\Theta} \subset \mathbb{R}^{q}$. In this setup, a popular estimation method is the following pseudo maximum likelihood estimation proposed in Genest, Ghoudi and Rivest [21].

The pseudo likelihood function, for $\boldsymbol{\theta}$ and pseudo observations $U_{i j}$ as in (3), is defined as

$$
L_{n}(\boldsymbol{\theta})=\prod_{i=1}^{n} c\left(U_{i 1}, \ldots, U_{i d} ; \boldsymbol{\theta}\right)
$$

which results in the pseudo maximum likelihood estimator $\hat{\boldsymbol{\theta}}_{n}=\arg \max L_{n}(\boldsymbol{\theta})$. The asymptotic behavior of the above pseudo maximum likelihood estimator and the estimator for the asymptotic variance are given in Genest, Ghoudi and Rivest [21]. When the marginals are also fitted to parametric families, the traditional maximum likelihood estimation is applied. A sieve maximum likelihood estimation is proposed by Chen, Fan and Tsyrennikov [7]. A comparison study on several different estimation procedures is given in Kim, Silvapulle and Silvapulle [34].

\subsection{Interval estimation}

Here we are interested in constructing a confidence interval for the copula $C\left(u_{1}, \ldots, u_{d}\right)$ at some particular point $\left(u_{1}, \ldots, u_{d}\right)^{\top} \in[0,1]^{d}$. An obvious approach is based on the asymptotic distribution of either nonparametric copula estimation or smooth copula estimation. Since the asymptotic variance of either estimate involves partial derivatives of the copula, it is required to either estimate the partial derivatives or using bootstrap methods. In order to avoid estimating the asymptotic variance, the empirical likelihood method has been employed recently; see Chen, Peng and Zhao [8] and Molanes Lopez, Van Keilegom and Veraverbeke [41]. As a nonparametric resampling method the empirical likelihood method is a powerful tool in interval estimation and hypothesis tests. We refer to Owen 
[43] for an overview on empirical likelihood methods. Recently, Jing, Yuan and Zhou [31] proposed a so-called jackknife empirical likelihood method to deal with non-linear functionals by applying the empirical likelihood method to some jackknife pseudo sample. This method is easy to implement. However, verifying Wilks's theorem (e.g. van der Vaart [58, Chapter 16]) requires some delicate analysis. Using the idea of jackknife empirical likelihood method, Peng, Qi and Van Keilegom [48] proposed a smoothed version to construct confidence intervals for a copula. We will explain this method for the bivariate case. Define for $\left(u_{1}, u_{2}\right)^{\top} \in[0,1]^{2}$

$$
\hat{\mathbb{C}}_{n, i}\left(u_{1}, u_{2}\right):=\frac{1}{n-1} \sum_{l=1, l \neq i}^{n} K_{1}\left(\frac{u_{1}-\mathbb{F}_{n 1, i}\left(X_{l 1}\right)}{h}\right) K_{1}\left(\frac{u_{2}-\mathbb{F}_{n 2, i}\left(X_{l 2}\right)}{h}\right)
$$

where $K_{1}(x):=\int_{-\infty}^{x} k_{1}(y) d y, k_{1}: \mathbb{R} \rightarrow \mathbb{R}_{0}^{+}$is a symmetric density function with support [-1,1], $h=h(n)>0$ is a bandwidth and $\mathbb{F}_{n j, i}(x)=\frac{1}{n-1} \sum_{l=1, l \neq i}^{n} \mathbb{1}\left(X_{l j} \leq x\right)$ for $j=1,2$. The smoothed jackknife pseudo sample is then defined for $i=1, \ldots, n$ as

$$
\mathbb{V}_{i}\left(u_{1}, u_{2}\right)=n \hat{\mathbb{C}}_{n}\left(u_{1}, u_{2}\right)-(n-1) \hat{\mathbb{C}}_{n, i}\left(u_{1}, u_{2}\right),
$$

where $\hat{\mathbb{C}}_{n}(\cdot, \cdot)$ is defined in (4) with $K_{2}(\cdot, \cdot)=K_{1}(\cdot) K_{1}(\cdot)$. Next we apply the empirical likelihood method based on estimating equations as in Qin and Lawless [52] to the above jackknife sample, which gives the jackknife empirical likelihood function for $\theta=C\left(u_{1}, u_{2}\right)$ as

$$
L_{n}(\theta)=\sup \left\{\prod_{i=1}^{n}\left(n p_{i}\right): p_{1} \geq 0, \ldots, p_{n} \geq 0, \sum_{i=1}^{n} p_{i}=1, \sum_{i=1}^{n} p_{i} \mathbb{V}_{i}\left(u_{1}, u_{2}\right)=\theta\right\} .
$$

Then Peng, Qi and Van Keilegom [48] showed the following version of Wilk's theorem:

$$
-2 \log L_{n}\left(\theta_{0}\right) \stackrel{d}{\longrightarrow} \chi_{1}^{2}
$$

as $n \rightarrow \infty$, where $\theta_{0}$ denotes the true value of $\theta=C\left(u_{1}, u_{2}\right)$. Based on this result, a confidence interval of level $1-\alpha \in(0,1)$ for $C\left(u_{1}, u_{2}\right)$ is given by

$$
I_{1-\alpha}^{C}\left(u_{1}, u_{2} ; h\right)=\left\{\theta:-2 \log L_{n}(\theta) \leq \chi_{1,1-\alpha}^{2}\right\},
$$

where $\chi_{1,1-\alpha}^{2}$ is the $(1-\alpha)$-quantile of $\chi_{1}^{2}$.

\subsection{Goodness-of-fit tests}

Here we want to test, whether the copula belongs to a particular parametric family. That is, we test

$$
H_{0}: C(\cdot) \in\{C(\cdot ; \boldsymbol{\theta}): \boldsymbol{\theta} \in \Theta\} \quad \text { against } \quad H_{1}: C(\cdot) \notin\{C(\cdot ; \boldsymbol{\theta}): \boldsymbol{\theta} \in \Theta\} .
$$


Based on a nonparametric estimator $\mathbb{C}_{n}(\cdot)$ and a parametric estimator $C\left(\cdot, \hat{\boldsymbol{\theta}}_{n}\right)$ of the copula, it is natural to consider rank-based versions of the Cramér-von Mises statistic

$$
\int_{[0,1]^{d}} n\left(\mathbb{C}_{n}(\mathbf{u})-C\left(\mathbf{u} ; \hat{\boldsymbol{\theta}}_{n}\right)\right)^{2} d \mathbb{C}_{n}(\mathbf{u}),
$$

or the Kolmogorov-Smirnov statistic

$$
\sup _{\mathbf{u} \in[0,1]^{d}}\left|\sqrt{n}\left(\mathbb{C}_{n}(\mathbf{u})-C\left(\mathbf{u} ; \hat{\boldsymbol{\theta}}_{n}\right)\right)\right|
$$

to perform the goodness-of-fit test. Tests based on Spearman's dependence function and smoothed copula estimates are given in Mesfioui, Quessy and Toupin [39] and Buchner and Dette [4], respectively. We also refer to Berg [3] and Genest, Remillard and Beaudoin [24] for overviews on goodness-of-fit testing for copulas.

\subsection{Empirical study: the Danish fire insurance data}

From the exploratory analysis in Section 1 we expect a dependence structure between the components of our data set. To check the null hypothesis of independence of losses to building and losses of contents, we perform an independence test; see e.g., Genest and Remillard [23]

$>$ indepTest(x.fire,empsamp)

Global Cramer-von Mises statistic: 0.3088693 with p-value 0.0004995005

Combined p-values from the M\"obius decomposition:

0.0004995005 from Fisher's rule,

0.0004995005 from Tippett's rule.

These $p$-values give strong evidence against the null hypothesis of independence. Therefore, it makes sense to consider different copula families to model the dependence between loss of building and loss of contents. To this end we perform goodness-of-fit tests for the Gumbel, Clayton, Frank, normal, and Plackett family. The parameters for the parametric fit are in each case computed by the pseudo maximum likelihood method (5). Only the Gumbel family is not rejected at the 0.05 level.

$>$ gofCopula( gumbel.cop, x.fire)

Parameter estimate(s): 1.254864

Cramer-von Mises statistic: 0.02323339 with p-value 0.2932068

The maximal $p$-value of the remaining four tests was 0.0185 . The left plot of Figure 2 shows the empirical copula $\mathbb{C}_{n}(x, y)$ together with fitted Gumbel and Clayton copula. The Clayton copula looses out on the Gumbel in particular in the tail region. 
Finally we also computed the standard error of the parameter estimate for the Gumbel copula by applying the function fitCopula() to the pseudo observations $U_{i j}$ from (3).

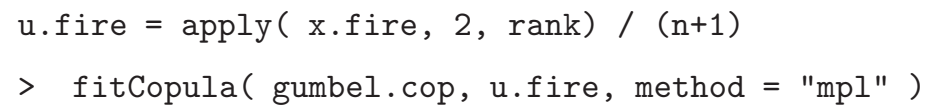

The estimation is based on the pseudo maximum likelihood

and a sample of size 301 .

Estimate Std. Error $\mathrm{z}$ value $\operatorname{Pr}(>|z|)$

param $1.2548640 .06396051 \quad 19.61936 \quad 0$

The maximized loglikelihood is 17.84647

The convergence code is 0

In Figure 2 we plot the empirical copula estimator $\mathbb{C}_{n}\left(u_{1}, 0.5\right)$ and the two jackknife confidence intervals $I_{0.90}^{C}\left(u_{1}, 0.5 ; 0.5 n^{-1 / 3}\right)$ and $I_{0.95}^{C}\left(u_{1}, 0.5 ; 0.5 n^{-1 / 3}\right)$.
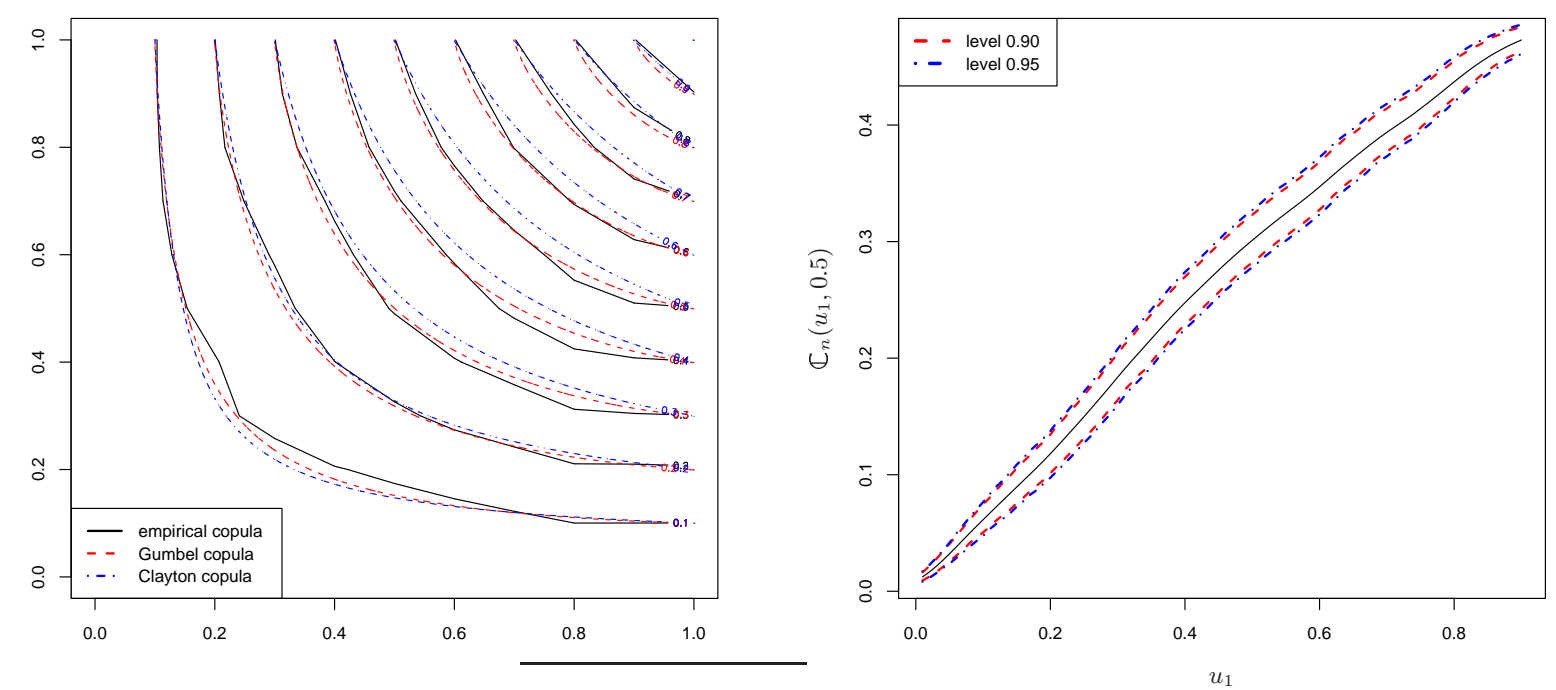

Figure 2. Left: Empirical (solid) copula $\mathbb{C}_{n}(x, y)$, fitted Gumbel (dashed) copula and fitted Clayton (dotted-dashed) copula. Plotted are the contour lines for levels $0.1,0.2, \ldots, 0.9$. Right: Empirical (solid) copula $\mathbb{C}_{n}\left(u_{1}, 0.5\right)$ with $90 \%$ (dashed) and $95 \%$ (dotted-dashed) jackknife confidence interval. 


\section{Inference for extreme value copulas}

\subsection{Point estimation}

Recently, Gudendorf and Segers [27] reviewed extreme value copulas and provided a list of many well-known parametric models. We recall some in the bivariate case, where we can use the following representation of a bivariate extreme value copula

$$
C\left(u_{1}, u_{2}\right)=\exp \left(\log \left(u_{1} u_{2}\right) A\left(\frac{\log \left(u_{2}\right)}{\log \left(u_{1} u_{2}\right)}\right)\right)
$$

for $\left(u_{1}, u_{2}\right)^{\top} \in[0,1]^{2}$, and $A$ is the so-called Pickands dependence function. It is easy to verify that $A$ is a convex function and satisfies $\max (t, 1-t) \leq A(t) \leq 1$ for all $t \in[0,1]$; see e.g., Beirlant et al. [1, Chapter 8.2.5]. In particular, we will give the parametric form of the Pickands dependence function $A(\cdot ; \boldsymbol{\theta})$ for the Gumbel, Galambos, Hüsler-Reiss, Tawn and $t$-EV copula for $t \in[0,1]$.

- Gumbel copula: for $\theta \in[1, \infty)$,

$$
A^{G u}(t)=\left(t^{\theta}+(1-t)^{\theta}\right)^{1 / \theta}
$$

- Galambos copula: for $\theta \in(0, \infty)$,

$$
A^{G a}(t ; \theta)=1-\left(t^{-\theta}+(1-t)^{-\theta}\right)^{-1 / \theta} .
$$

- Hüsler-Reiss copula: for $\theta \in[0, \infty]$,

$$
A^{H R}(t ; \theta)=(1-t) \Phi\left(\theta+\frac{1}{2 \theta} \log \left(\frac{1-t}{t}\right)\right)+t \Phi\left(\theta+\frac{1}{2 \theta} \log \left(\frac{t}{1-t}\right)\right) .
$$

- Tawn copula: for $\theta \in[0,1]$,

$$
A(t ; \theta)=1-\theta t+\theta t^{2} .
$$

- $t$-EV copula: for $\boldsymbol{\theta}=\left(\theta_{1}, \theta_{2}\right) \in(0, \infty) \times(-1,1)$,

$$
A^{t-E V}(t ; \boldsymbol{\theta})=t t_{\theta_{1}+1}\left(\frac{\left(\frac{t}{1-t}\right)^{1 / \theta_{1}}-\theta_{2}}{\sqrt{1-\theta_{2}^{2}}} \sqrt{\theta_{1}+1}\right)+(1-t) t_{\theta_{1}+1}\left(\frac{\left(\frac{1-t}{t}\right)^{1 / \theta_{1}}-\theta_{2}}{\sqrt{1-\theta_{2}^{2}}} \sqrt{\theta_{1}+1}\right),
$$

with $t_{\theta}$ representing the distribution function of the $t_{\theta}$ distribution.

For parametric estimation, methods for copulas can be employed directly such as the pseudo maximum likelihood estimation (5); see e.g., Beirlant et al. [1, Chapter 9.3.2] and references therein. 
Here we will focus on nonparametric estimation methods, which are different from the nonparametric methods for copulas, since every estimation method has to guarantee that the estimated copula is an extreme value copula; i.e., it satisfies (1).

Let $\left(X_{11}, X_{12}\right), \ldots,\left(X_{n 1}, X_{n 2}\right)$ be independent random vectors with common distribution function $F$. Assume that the copula $C$ of $F$ is an extreme value copula; i.e., that (1) holds.

When the marginals are known, several estimators have been proposed in the literature and the corresponding asymptotic distributions have been derived as well, we refer to Pickands [50], Deheuvels [11], Capéraà, Fourgeres and Genest [5], Hall and Tajvidi [28], and Zhang, Wells and Peng [59]. However, when the marginals in the above estimators are replaced by empirical distribution functions, how to derive the asymptotic distributions, has remained unknown till 2009. More specifically, Genest and Segers [25] derived the asymptotic distributions of the estimators proposed by Pickands [50] and Capéraà, Fourgeres and Genest [5], respectively,

$$
\mathbb{A}_{n}^{P}(t)=\left(\frac{1}{n} \sum_{i=1}^{n} \frac{-\log \left(U_{i 1}\right)}{1-t} \wedge \frac{-\log \left(U_{i 2}\right)}{t}\right)^{-1}
$$

and

$$
\mathbb{A}_{n}^{C F G}(t)=\exp \left(-\gamma-\frac{1}{n} \sum_{i=1}^{n} \log \left(\frac{-\log \left(U_{i 1}\right)}{1-t} \wedge \frac{-\log \left(U_{i 2}\right)}{t}\right)\right),
$$

for $t \in[0,1]$, where $\gamma=-\int_{0}^{\infty} \log (u) \exp (-u) d u$ is Euler's constant. They realized that these estimators can be expressed as functionals of the empirical copula; more precisely, for $t \in[0,1]$,

$$
\mathbb{A}_{n}^{P}(t)=\left(\int_{0}^{1} \mathbb{C}_{n}\left(u^{1-t}, u^{t}\right) u^{-1} d u\right)^{-1}
$$

and

$$
\mathbb{A}_{n}^{C F G}(t)=\exp \left(-\gamma+\int_{0}^{1} \frac{\mathbb{C}_{n}\left(u^{1-t}, u^{t}\right)-\mathbb{1}\left(u>e^{-1}\right)}{u \log (u)} d u\right)
$$

which allows us to use a result of Rüschendorf [54] for the empirical copula process $\sqrt{n}\left(\mathbb{C}_{n}-C\right)$.

Recently, Peng, Qian and Yang [49] proposed a class of weighted estimators by solving the following equation with respect to $\alpha \geq 0$ :

$$
\int_{0}^{1}\left\{\mathbb{C}_{n}\left(u^{1-t}, u^{t}\right)-u^{\alpha}\right\} \lambda(u, t) d u=0
$$

for some weight function $\lambda(u, t) \geq 0$. Then the above two estimators in (8) and (9) correspond to $\lambda(u, t)=u^{-1}$ and $\{-u \log (u)\}^{-1}$, respectively. The asymptotic distributions for the above weighted estimators are as well derived in Peng, Qian and Yang [49] by using the weighted approximation of empirical copula processes. 


\subsection{Interval estimation}

In order to construct confidence intervals for the Pickands dependence function $A$, it is possible to employ the normal approximation method based on one of the nonparametric estimators for $A$. Since the asymptotic variance involves the first derivative $A^{\prime}$ of $A$, Genest and Segers [25] proposed a way to estimate $A^{\prime}$ and the asymptotic variance. Instead of this rather involved method, a naive bootstrap method can be applied to construct confidence intervals for $A(t)$. It is known that these types of intervals have poor coverage accuracy in general. As an alternative way for interval estimation without estimating the asymptotic variance explicitly, the empirical likelihood method has been applied successfully. Although the empirical likelihood method works well for linear functionals, some transformation is needed before applying profile empirical likelihood methods to nonlinear functionals. Hence this procedure increases the computational burden in general. Using the idea of the jackknife empirical likelihood method, Peng, Qian and Yang [49] proposed a smoothed version to construct confidence intervals for the Pickands dependence function. The details are as follows. Define for the pseudo observations $U_{i j}$ as in (3)

$$
\mathbb{C}_{n}\left(u^{1-t}, u^{t}\right):=\frac{1}{n} \sum_{i=1}^{n} K_{1}\left(\frac{u-U_{i 1}^{1 /(1-t)}}{h}\right) K_{1}\left(\frac{u-U_{i 2}^{1 / t}}{h}\right), \quad u \in[0,1],
$$

where $K_{1}$ is defined in Section 2.2 and $h=h(n)>0$ is again a bandwidth. For $j=1,2$ and $k, i=$ $1, \ldots, n$ set $U_{k j, i}=n^{-1} \sum_{l=1, l \neq i}^{n} \mathbb{1}\left(X_{l j} \leq X_{k j}\right)$ and

$$
\mathbb{C}_{n, i}\left(u^{1-t}, u^{t}\right):=\frac{1}{n-1} \sum_{l=1, l \neq i}^{n} K_{1}\left(\frac{u-U_{l 1, i}^{1 /(1-t)}}{h}\right) K_{1}\left(\frac{u-U_{l 2, i}^{1 / t}}{h}\right) .
$$

We formulate the jackknife sample as

$$
\mathbb{V}_{i}(u, t)=n \mathbb{C}_{n}\left(u^{1-t}, u^{t}\right)-(n-1) \mathbb{C}_{n, i}\left(u^{1-t}, u^{t}\right), \quad u, t \in[0,1],
$$

for $i=1, \ldots, n$. Next we apply the empirical likelihood method based on estimating equations as in Qin and Lawless [52] to the above jackknife sample, which gives the jackknife empirical likelihood function for $\theta=A(t)$ as

$$
L_{n}(\theta)=\sup \left\{\prod_{i=1}^{n}\left(n p_{i}\right): p_{1} \geq 0, \ldots, p_{n} \geq 0, \sum_{i=1}^{n} p_{i}=1, \sum_{i=1}^{n} p_{i} \int_{a_{n}}^{1-b_{n}}\left\{\mathbb{V}_{i}(u, t)-u^{\theta}\right\} \lambda(u, t) d u=0\right\},
$$

where $a_{n}>0$ and $b_{n}>0$. The reason to employ $a_{n}$ and $b_{n}$ is to control the bias term in the smoothed estimation and to allow for the possibility of $\lambda(0, t)=\infty$ and $\lambda(1, t)=\infty$. Then Peng, Qian and Yang [49] showed the following version of Wilks's theorem. 
Theorem 1. With the notation as above, suppose that the second derivative $A^{\prime \prime}(t)$ is continuous on $[0,1]$ and

$$
\left\{\begin{array}{l}
h=h(n) \rightarrow 0, n h \rightarrow \infty, \\
a_{n} \rightarrow a \in[0,1 / 2), b_{n} \rightarrow b \in[0,1 / 2), h / a_{n} \rightarrow 0, h / b_{n} \rightarrow 0, \\
n^{-1 / 4+\delta_{1}} \int_{a_{n}}^{1-b_{n}} \lambda(u, t) d u \rightarrow 0, \\
\int_{0}^{1} u^{\delta_{2}}\left(1-u^{\delta_{2}}\right) \lambda(u, t) d u<\infty, \\
\sqrt{n} h^{2} \int_{a_{n}}^{1-b_{n}} u^{-3 / 2} \lambda(u, t) d u \rightarrow 0, \\
\sqrt{n} h^{2} \int_{a_{n}}^{1-b_{n}}\{\log u\}^{-1} u^{-3 / 2} \lambda(u, t) d u \rightarrow 0, \\
\{\sqrt{n} h\}^{-1} \int_{a_{n}}^{1-b_{n}} u^{-1} \lambda(u, t) d u \rightarrow 0, \\
n^{-3 / 2} \int_{a_{n}}^{1-b_{n}} u^{-2} \lambda(u, t) d u \rightarrow 0
\end{array}\right.
$$

as $n \rightarrow \infty$. Then the pseudo likelihood function as defined in (5) satisfies for $t \in(0,1)$,

$$
-2 \log L_{n}\left(\theta_{0}\right) \stackrel{d}{\longrightarrow} \chi_{1}^{2},
$$

as $n \rightarrow \infty$, where $\theta_{0}$ denotes the true value of $\theta=A(t)$ and $\chi_{1}^{2}$ the $\chi_{1}^{2}$-distribution.

Based on the above theorem, a jackknife empirical likelihood confidence interval for $A(t)$ with level $1-\alpha \in(0,1)$ can be constructed as

$$
I_{1-\alpha}^{A}(t ; h)=\left\{\theta:-2 \log L_{n}(\theta) \leq \chi_{1,1-\alpha}^{2}\right\},
$$

where $\chi_{1,1-\alpha}^{2}$ is the $(1-\alpha)$-quantile of the $\chi_{1}^{2}$ distribution.

\subsection{Goodness-of-fit tests}

For testing whether the extreme-value copula comes from a particular parametric class, we compare the distance between nonparametric and parametric estimation of the extreme value copula as usual. Unlike the study of goodness-of-fit tests for copulas, results on goodness-of-fit tests for extremevalue copulas are relatively rare in the literature; we mention Ghoudi, Khoudraji and Rivest [26], Ben Ghorbal, Genest and Nešlehová [2], and the recent results of Genest et al. [22]. We derive some Cramér-von Mises type test based on the weighted estimator (10) of the Pickands dependence function suggested in Peng, Qian and Yang [49].

Suppose we want to test (6) and the copula is an extreme value copula; i.e., (7) holds. Hence, for some $\boldsymbol{\theta} \in \Theta$

$$
C\left(u_{1}, u_{2} ; \boldsymbol{\theta}\right)=\exp \left(\log \left(u_{1} u_{2}\right) A\left(\frac{\log \left(u_{2}\right)}{\log \left(u_{1} u_{2}\right)} ; \boldsymbol{\theta}\right)\right), \quad\left(u_{1}, u_{2}\right)^{\top} \in[0,1]^{2}
$$


Let $\hat{A}^{\lambda}(t)$ denote the weighted estimation of Pickands dependence function; i.e., $\hat{A}^{\lambda}(t)$ is the solution to (10). Hence $C\left(u_{1}, u_{2}\right)$ is estimated by

$$
\hat{C}_{n}^{\lambda}\left(u_{1}, u_{2}\right)=\exp \left(\log \left(u_{1} u_{2}\right) \hat{A}^{\lambda}\left(\frac{\log \left(u_{2}\right)}{\log \left(u_{2} u_{2}\right)}\right)\right) .
$$

Further let $\hat{\boldsymbol{\theta}}$ denote the pseudo maximum likelihood estimate of $\boldsymbol{\theta}$ as in (5). Some type of Cramér-von Mises test statistic is defined as

$$
T_{C M}=n \int_{0}^{1} \int_{0}^{1}\left\{\hat{C}_{n}^{\lambda}\left(u_{1}, u_{2}\right)-C\left(u_{1}, u_{2} ; \hat{\boldsymbol{\theta}}\right)\right\}^{2} d C\left(u_{1}, u_{2} ; \hat{\boldsymbol{\theta}}\right),
$$

and its asymptotic distributions is given in the following theorem. Other types of tests based on the distance between $\hat{C}_{n}^{\lambda}\left(u_{1}, u_{2}\right)$ and $C\left(u_{1}, u_{2} ; \hat{\boldsymbol{\theta}}\right)$ can be derived similarly. Set

$l\left(u_{1}, u_{2} ; \boldsymbol{\theta}\right)=\log \left(\frac{\partial^{2}}{\partial u_{1} \partial u_{2}} C\left(u_{1}, u_{2} ; \boldsymbol{\theta}\right)\right), \quad i\left(u_{1}, u_{2} ; \boldsymbol{\theta}\right)=\frac{\partial}{\partial \boldsymbol{\theta}} l\left(u_{1}, u_{2} ; \boldsymbol{\theta}\right), \quad \ddot{l}\left(u_{1}, u_{2} ; \boldsymbol{\theta}\right)=\frac{\partial^{2}}{\partial \boldsymbol{\theta} \partial \boldsymbol{\theta}^{\top}} l\left(u_{1}, u_{2} ; \boldsymbol{\theta}\right)$,

$$
i_{12}\left(u_{1}, u_{2} ; \boldsymbol{\theta}\right)=\frac{\partial^{2}}{\partial u_{1} \partial u_{2}} \dot{l}\left(u_{1}, u_{2} ; \boldsymbol{\theta}\right), \quad \dot{C}\left(u_{1}, u_{2} ; \boldsymbol{\theta}\right)=\frac{\partial}{\partial \boldsymbol{\theta}} C\left(u_{1}, u_{2} ; \boldsymbol{\theta}\right),
$$

$C_{1}\left(u_{1}, u_{2} ; \boldsymbol{\theta}\right)=\frac{\partial}{\partial u_{1}} C\left(u_{1}, u_{2} ; \boldsymbol{\theta}\right), \quad C_{2}\left(u_{1}, u_{2} ; \boldsymbol{\theta}\right)=\frac{\partial}{\partial u_{2}} C\left(u_{1}, u_{2} ; \boldsymbol{\theta}\right), \quad \Sigma=\mathbb{E}\left(\ddot{l}\left(F_{1}\left(X_{11}\right), F_{2}\left(X_{12}\right) ; \boldsymbol{\theta}_{0}\right)\right)$, where $\boldsymbol{\theta}_{0}$ denotes the true value of $\boldsymbol{\theta}$.

Theorem 2. Suppose that the second derivative $A^{\prime \prime}(t)$ is continuous on $[0,1]$ and

$$
\left\{\begin{array}{l}
\sup _{0 \leq t \leq 1} \sqrt{n} \int_{0}^{(n+1)^{-1 /((1-t) \vee t)}} u^{1 / 2} \lambda(u, t) d u \rightarrow 0, \\
\sup _{0 \leq t \leq 1} \sqrt{n} \int_{\left(\frac{n}{n+1}\right)^{1 /((1-t) \vee t)}}^{1}(1-u) \lambda(u, t) d u \rightarrow 0, \\
\sup _{0 \leq t \leq 1} n^{-1 / 4+\delta_{1}} \int_{(n+1)^{-1 /((1-t) \vee t)}}^{\left(\frac{n}{n+1}\right)^{1 /((1-t) \vee)} \lambda(u, t) d u \rightarrow 0,} \\
\sup _{0 \leq t \leq 1} \int_{0}^{1}\left\{u^{(1-t) \vee t}\left(1-u^{(1-t) \vee t}\right)\right\}^{\delta_{2}} \lambda(u, t) d u<\infty, \\
\sup _{0 \leq t \leq 1} \int_{0}^{1} u^{(1-t) \vee t-(1-t)}\left(1-u^{1-t}\right)^{\delta_{2}} \lambda(u, t) d u<\infty, \\
\sup _{0 \leq t \leq 1} \int_{0}^{1} u^{(1-t) \vee t-t} u^{t \delta_{2}}\left(1-u^{t}\right)^{\delta_{2}} \lambda(u, t) d u<\infty, \\
\sup _{0 \leq t \leq 1} \int_{1 / 2}^{1}(-\log u) \lambda(u, t) d u<\infty
\end{array}\right.
$$

for some $\lambda(u, t) \geq 0, \delta_{1}>0$ and $\delta_{2} \in[0,1 / 2)$. Further assume that there exist $\delta \in(0,1 / 2)$, some nonnegative functions $M_{1}\left(u_{1}, u_{2}\right), M_{2}\left(u_{1}, u_{2}\right)$, and an open neighborhood $N\left(\boldsymbol{\theta}_{0}\right)$ of $\boldsymbol{\theta}_{0}$ such that

$$
\left\{\begin{array}{l}
\int_{0}^{1} \int_{0}^{1}\left(u_{1} \wedge u_{2}\right)^{\delta}\left(1-u_{1} \wedge u_{2}\right)^{\delta}\left|\dot{l}_{12}\left(u_{1}, u_{2} ; \boldsymbol{\theta}_{0}\right)\right| d u_{1} d u_{2}<\infty, \\
\int_{0}^{1} \int_{0}^{1} u_{i}^{\delta}\left(1-u_{i}\right)^{\delta} C_{i}\left(u_{1}, u_{2} ; \boldsymbol{\theta}_{0}\right)\left|\dot{l}_{12}\left(u_{1}, u_{2} ; \boldsymbol{\theta}_{0}\right)\right| d u_{1} d u_{2}<\infty, \quad \text { for } i=1,2, \\
\int_{0}^{1} \int_{0}^{1} \sup _{\boldsymbol{\theta} \in N\left(\boldsymbol{\theta}_{0}\right)}\left|\ddot{l}\left(u_{1}, u_{2} ; \boldsymbol{\theta}\right)\right| d C\left(u_{1}, u_{2} ; \boldsymbol{\theta}_{0}\right)<\infty, \\
\sup _{\boldsymbol{\theta} \in N\left(\boldsymbol{\theta}_{0}\right)}\left|\ddot{l}\left(u_{1}, u_{2} ; \boldsymbol{\theta}\right)-\ddot{l}\left(v_{1}, v_{2} ; \boldsymbol{\theta}\right)\right| \leq\left|u_{1}-v_{1}\right| M_{1}\left(u_{1}, u_{2}\right)+\left|u_{2}-v_{2}\right| M_{2}\left(v_{1}, v_{2}\right), \\
\int_{0}^{1} \int_{0}^{1} u_{i}^{\delta} M_{i}\left(u_{1}, u_{2}\right) d C\left(u_{1}, u_{2} ; \boldsymbol{\theta}_{0}\right)<\infty, \quad \text { for } i=1,2,
\end{array}\right.
$$


and $\Sigma^{-1}$ exists. Then

$$
T_{C M} \stackrel{d}{\longrightarrow} \int_{0}^{1} \int_{0}^{1}\left\{C\left(u_{1}, u_{2} ; \boldsymbol{\theta}_{0}\right) \log \left(u_{1} u_{2}\right) B\left(\frac{\log u_{2}}{\log \left(u_{1} u_{2}\right)}\right)+B^{*} \Sigma^{-1} \dot{C}\left(u_{1}, u_{2} ; \boldsymbol{\theta}_{0}\right)\right\}^{2} d C\left(u_{1}, u_{2} ; \boldsymbol{\theta}_{0}\right),
$$

where

$$
\begin{aligned}
B^{*} & =\int_{0}^{1} \int_{0}^{1}\left\{W\left(u_{1}, u_{2}\right)-C_{1}\left(u_{1}, u_{2} ; \boldsymbol{\theta}_{0}\right) W\left(u_{1}, 1\right)-C_{2}\left(u_{1}, u_{2} ; \boldsymbol{\theta}_{0}\right) W\left(1, u_{2}\right)\right\} i_{12}^{\top}\left(u_{1}, u_{2} ; \boldsymbol{\theta}_{0}\right) d u_{1} d u_{2}, \\
B(t) & =\frac{\int_{0}^{1}\left\{W\left(u^{1-t}, u^{t}\right)-C_{1}\left(u^{1-t}, u^{t}\right) W\left(u^{1-t}, 1\right)-C_{2}\left(u^{1-t}, u^{t}\right) W\left(1, u^{t}\right)\right\} \lambda(u, t) d u}{\int_{0}^{1} C\left(u^{1-t}, u^{t}\right) \lambda(u, t) \log (u) d u},
\end{aligned}
$$

and $W\left(u_{1}, u_{2}\right)$ is a Wiener process with zero mean and covariance

$$
\mathbb{E}\left(W\left(u_{1}, u_{2}\right) W\left(v_{1}, v_{2}\right)\right)=C\left(u_{1} \wedge v_{1}, u_{2} \wedge v_{2}\right)-C\left(u_{1}, u_{2}\right) C\left(v_{1}, v_{2}\right) .
$$

Proof. It follows from Peng, Qian and Yang [49] that

$$
\sup _{0 \leq t \leq 1}\left|\sqrt{n}\left\{\hat{A}^{\lambda}(t)-A(t)\right\}-B(t)\right|=o_{p}(1) .
$$

Using similar arguments as in Chan et al. [6], we can show that

$$
\sqrt{n}\{\hat{\boldsymbol{\theta}}-\boldsymbol{\theta}\} \stackrel{d}{\longrightarrow}-\Sigma^{-1} B^{* \top} .
$$

Write

$$
\hat{C}_{n}^{\lambda}(u, v)-C(u, v ; \hat{\boldsymbol{\theta}})=\hat{C}_{n}^{\lambda}(u, v)-C\left(u, v ; \boldsymbol{\theta}_{0}\right)-\left\{C(u, v ; \hat{\boldsymbol{\theta}})-C\left(u, v ; \boldsymbol{\theta}_{0}\right)\right\} .
$$

Then the theorem follows from a Taylor expansion and the above equations.

\subsection{The Danish fire insurance data (continued)}

In Section 2.4 the Gumbel family was the only copula family under consideration, which was not rejected by a Cramér-von Mises type goodness-of-fit test. Since the Gumbel copula is not only an Archimedian copula, but also an extreme value copula, it is already a valid candidate in this framework.

Our data consists of claims exceeding one million Danish Kroner. Therefore, it makes sense to consider extreme value copulas to describe the dependence. The rank-based goodness-of-fit test introduced in Genest et al. [22] can be performed by applying the function gofEVCopula() from the copula package. The function returns the test statistics based on the empirical copula (this is the same result as obtained from gofCopula()) and the corrected and uncorrected estimators of $A$ introduced in Pickands [50] and Capéraà, Fourgeres and Genest [5]. As extreme value copulas we consider the Gumbel, Galambos, Hüsler-Reiss, Tawn and $t$-EV copula. The highest $p$-values for all five tests are obtained for the Hüsler-Reiss family. 
$>$ gofEVCopula( huslerReiss.cop, x.fire)

\$statistic

[1] $0.01918947 \quad 0.03430994 \quad 0.04269746 \quad 0.01763666 \quad 0.02585871$

\$pvalue

[1] $0.5029970 \quad 0.0984016 \quad 0.1013986 \quad 0.8806194 \quad 0.8986014$

\$parameters

[1] 0.904332

The test based on the Capéraà-Fougéres-Genest estimator rejected the null hypothesis of a Tawn copula at the 0.05 level. All other null hypothesis were not rejected at this level. Finally we compare the maximized likelihood of the Gumbel copula model with the one from the Hüsler-Reiss copula.

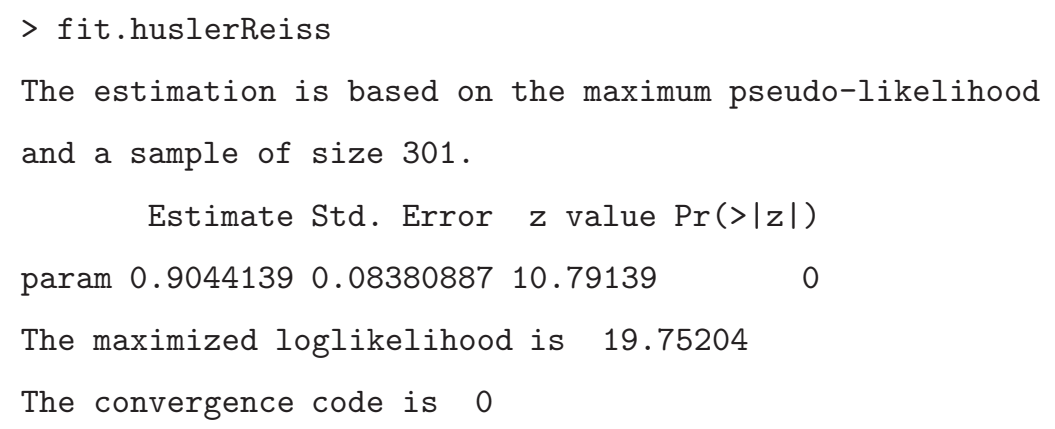

The higher likelihood is obtained for the Hüsler-Reiss copula. Therefore we would choose this copula, if we had to decide between the two of them. In Figure 3 we compare the fitted copulas visually and can recognize no difference.

\section{Inference for tail copulas}

Throughout we focus on the case of $d=2$.

\subsection{Point estimation}

A natural nonparametric estimator for the tail copula $T$ at $\left(x_{1}, x_{2}\right)^{\top} \in(0, \infty)^{2}$ defined in (2) is

$$
\mathbb{T}\left(x_{1}, x_{2} ; m\right)=\frac{1}{m} \sum_{i=1}^{n} \mathbb{1}\left(\mathbb{F}_{n 1}\left(X_{i 1}\right)>1-\frac{m}{n} x_{1}, \mathbb{F}_{n 2}\left(X_{i 2}\right)>1-\frac{m}{n} x_{2}\right),
$$

where $m=m(n) \rightarrow \infty$ and $m / n \rightarrow 0$ as $n \rightarrow \infty$. For ways of choosing the threshold $m$ in (11) we refer to Schmidt and Stadtmüller [56] and Klüppelberg, Kuhn and Peng [36]. Note that every estimator for the tail dependence function (see Huang [30]) or the spectral measure (see de Haan and Ferreira [9]) 

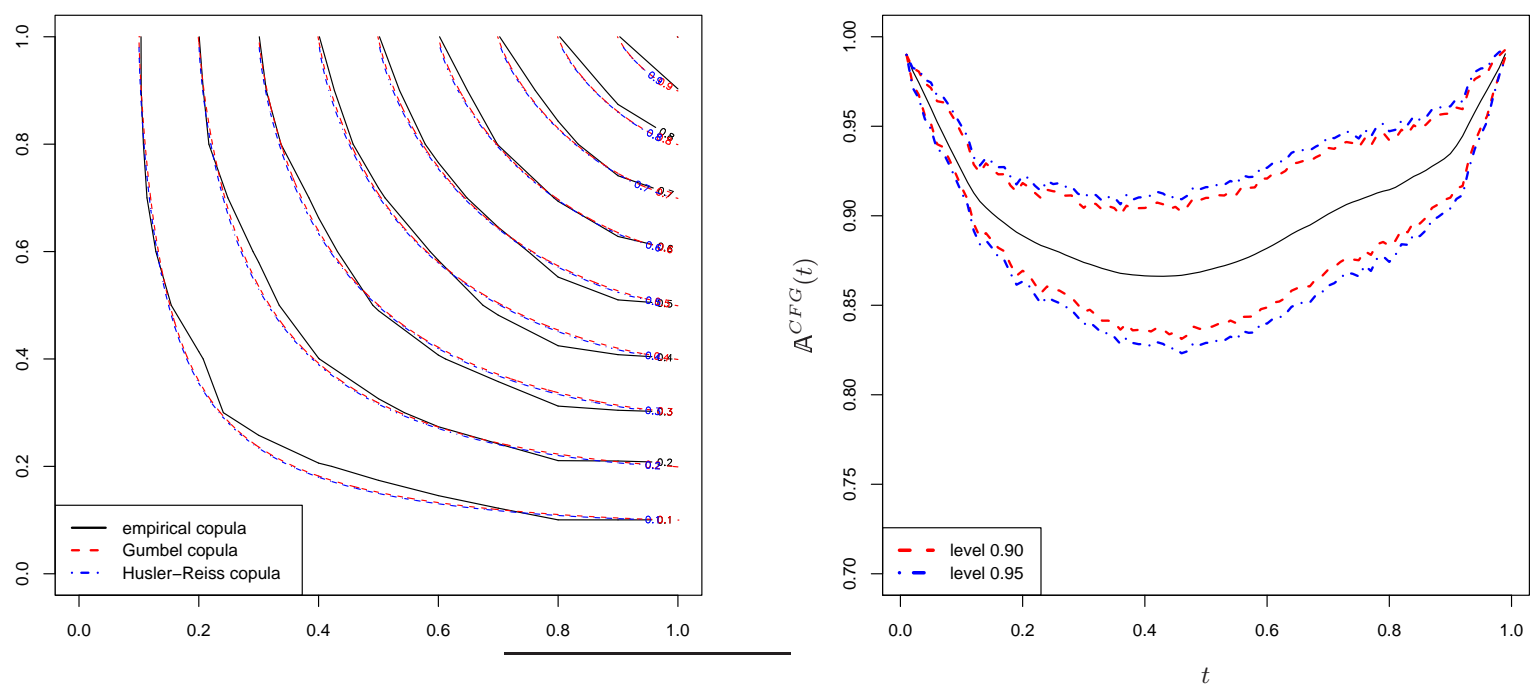

FiguRE 3. Left panel plots the empirical (solid) copula $\mathbb{C}_{n}(x, y)$, fitted Gumbel (dashed) copula and fitted Hüsler-Reiss (dotted-dashed) copula. Right panel shows the $90 \%$ and $95 \%$ confidence intervals based on the estimator $\mathbb{A}^{C F G}(t)$.

can be transformed to an estimator for the tail copula. For the proof of consistency, the asymptotic distribution and weighted approximations of $\mathbb{T}\left(x_{1}, x_{2} ; m\right)$, we refer to Huang [30], Qi [51], Drees and Huang [12], Einmahl, de Haan and Li [13], and Schmidt and Stadtmüller [56]. Note that T(1,1) is the upper tail dependence coefficient, which is often used as a simple measure for extreme dependence (cf. Joe [32]). Hence, $\mathbb{T}(1,1 ; m)$ is the empirical estimator for $T(1,1)$.

Some parametric models for the tail copula can be found in Tawn [57]. Recently, a parametric class of tail copulas is constructed via elliptical copulas; see Klüppelberg, Kuhn and Peng [35]. More specifically, let $\mathbf{Z}:=G \mathcal{M} \mathbf{U}^{(2)}$ be an elliptically distributed random vector, where $G>0$ is a positive random variable satisfying $\lim _{t \rightarrow \infty} P(G>t x) / P(G>t)=x^{-\nu}$ for all $x>0$ and some $\nu>0$, $\mathbf{U}^{(2)} \sim \operatorname{unif}\left(\left\{\left(x_{1}, x_{2}\right) \in \mathbb{R}^{2}: x_{1}^{2}+x_{2}^{2}=1\right\}\right)$ is independent of $G$ and $\mathcal{M}$ is a deterministic $2 \times 2$ matrix with $\boldsymbol{M M}^{\top}=\left(\begin{array}{ll}1 & \rho \\ \rho & 1\end{array}\right)$. Then the tail copula of $\mathbf{Z}$ has the following explicit expression

$$
T\left(x_{1}, x_{2} ;(\nu, \rho)\right)=\left(x_{1} \int_{g\left(\left(x_{1} / x_{2}\right)^{1 / \nu}\right.}^{\pi / 2}(\cos \phi)^{\nu} d \phi+x_{2} \int_{g\left(\left(x_{1} / x_{2}\right)^{-1 / \nu}\right)}^{\pi / 2}(\cos \phi)^{\nu} d \phi\right)\left(\int_{-\pi / 2}^{\pi / 2}(\cos \phi)^{\nu} d \phi\right)^{-1},
$$

where $g(t):=\arctan \left((t-\rho) / \sqrt{1-\rho^{2}}\right)$.

When the tail copula is fitted by a parametric class, maximum likelihood estimators, moment estimators and their asymptotic distributions are developed in de Haan, Neves and Peng [10] and 
Einmahl, Krajina and Segers [14], respectively. In Klüppelberg, Kuhn and Peng [35, 36] an estimator is proposed for the parameter $\nu$ of an elliptical tail copula. They apply the relation between Kendall's tau and the correlation coefficient for elliptical tail copulas, which translates to the estimators

$$
\hat{\rho}_{n}:=\sin \left(\frac{\pi}{2} \hat{\tau}_{n}\right),
$$

where $\hat{\tau}_{n}$ is a rank-based estimator of Kendall's tau. They use this estimator as a plug-in-estimator for $\rho$ in the inverse $T^{-1}\left(\cdot \mid x_{1}, x_{2}, \rho\right)$ of $T$ with respect to $\nu$. The estimator for $\nu$ is then defined as

$$
\hat{\nu}_{n}=T^{-1}\left(\mathbb{T}\left(x_{1}, x_{2} ; m\right) \mid x_{1}, x_{2}, \hat{\rho}_{n}\right) .
$$

\subsection{Interval estimation}

When the tail copula is modeled by a parametric family, interval estimation for the tail copula at a given point $\left(x_{1}, x_{2}\right)$ can be obtained via the normal approximation method. Alternatively one can use methods based on nonparametric estimation and bootstrap as developed in Peng and Qi [45, 46]. Recently, a smoothed jackknife empirical likelihood method has been proposed by Peng and Qi [47] to construct a confidence interval for the tail copula without estimating the asymptotic variance. We review it in some detail. Define the smoothed tail copula estimators

$$
\hat{\mathbb{T}}_{n}\left(x_{1}, x_{2} ; m, h\right)=\frac{1}{n} \sum_{l=1}^{n} K_{1}\left(\frac{x_{1}-n\left(1-\mathbb{F}_{n 1}\left(X_{l 1}\right)\right) / m}{h}\right) K_{1}\left(\frac{x_{2}-n\left(1-\mathbb{F}_{n 2}\left(X_{l 2}\right)\right) / m}{h}\right)
$$

and

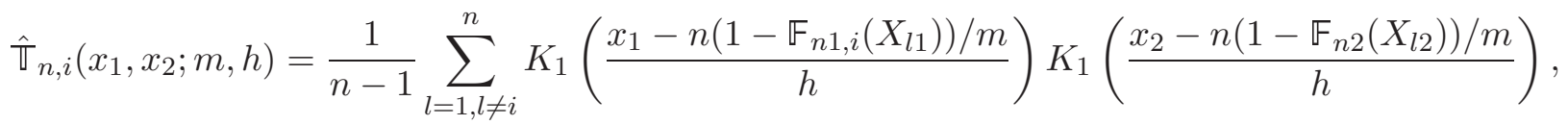

where $K_{1}$ is defined in Section 2.2 and $h=h(n)>0$ is again a bandwidth. Further define the jackknife sample as

$$
\mathbb{V}_{i}\left(x_{1}, x_{2} ; h\right)=n \hat{\mathbb{\mathbb { V }}}_{n}\left(x_{1}, x_{2} ; m, h\right)-(n-1) \hat{\mathbb{T}}_{n, i}\left(x_{1}, x_{2} ; m, h\right) \quad \text { for } \quad i=1, \ldots, n
$$

Based on the above jackknife sample, one can define the smoothed jackknife empirical likelihood function for $\theta=T\left(x_{1}, x_{2}\right)$ as

$$
L_{n}\left(x_{1}, x_{2} ; \theta\right)=\sup \left\{\prod_{i=1}^{n}\left(n p_{i}\right): p_{1} \geq 0, \ldots, p_{n} \geq 0, \sum_{i=1}^{n} p_{i}=1, \sum_{i=1}^{n} p_{i} \mathbb{V}_{i}\left(x_{1}, x_{2} ; h\right)=\frac{m}{n} \theta\right\} .
$$

In order to show that Wilks's theorem holds for the proposed method, we need some regularity conditions. Then the following theorem follows from Peng and Qi [47]. 
Theorem 3. With the notation as above we assume that $k_{1}$ is a symmetric density with support $[-1,1]$ and has bounded first derivative. We assume also that

$$
\left\{\begin{array}{l}
m=m(n) \rightarrow \infty, \quad m / n \rightarrow 0, \quad \sqrt{m} a(m / n) \rightarrow 0 \text { as } n \rightarrow \infty \\
h=h(n) \rightarrow 0, \quad m h^{2} \rightarrow \infty, \quad m h^{4} \rightarrow 0 \text { as } n \rightarrow \infty .
\end{array}\right.
$$

Furthermore, we assume that $T\left(x_{1}, x_{2}\right)$ has continuous first partial derivatives and that there exists a regularly varying function $a(t) \rightarrow 0$ such that

$$
\lim _{t \rightarrow 0} \frac{t^{-1} P\left(F_{1}\left(X_{11}\right)>1-t x_{1}, F_{2}\left(X_{12}\right)>1-t x_{2}\right)-T\left(x_{1}, x_{2}\right)}{a(t)}=d\left(x_{1}, x_{2}\right)
$$

holds uniformly on the set $\left\{\left(x_{1}, x_{2}\right): x_{1} \geq 0, x_{2} \geq 0, x_{1}^{2}+x_{2}^{2}=1\right\}$, where $d\left(x_{1}, x_{2}\right)$ is non-constant and not a multiple of $T\left(x_{1}, x_{2}\right)$. Then

$$
-2 \log L_{n}\left(x_{1}, x_{2} ; \theta_{0}\right) \stackrel{d}{\longrightarrow} \chi_{1}^{2}
$$

as $n \rightarrow \infty$, where $\theta_{0}$ denotes the true value of $\theta=T\left(x_{1}, x_{2}\right)$.

As in Section 3.2, a jackknife empirical likelihood confidence interval for $T(x, y)$ with level $1-\alpha \in$ $(0,1)$ can be constructed as

$$
I_{1-\alpha}^{T}\left(x_{1}, x_{2} ; m, h\right)=\left\{\theta:-2 \log L_{n}\left(x_{1}, x_{2} ; \theta\right) \leq \chi_{1,1-\alpha}^{2}\right\}
$$

where $\chi_{1,1-\alpha}^{2}$ is the $(1-\alpha)$-quantile of the $\chi_{1}^{2}$-distribution.

\subsection{Goodness-of-fit tests}

For testing whether a tail copula belongs to a particular parametric family, de Haan, Neves and Peng [10] proposed tests based on the distance between a nonparametric estimator and the maximum likelihood estimator of the tail copula. Tests based on a moment estimator are given in Einmahl, Krajina and Segers [14]. In case the data $\mathbf{X}$ has an elliptical copula, Li and Peng [38] proposed a test based on estimators in Klüppelberg, Kuhn and Peng [36]. How to derive the empirical likelihood ratio test remains still unknown.

\subsection{The Danish fire insurance data (continued)}

Finally we want to consider the extreme dependence in the losses to building and contents. We start by estimating the upper tail dependence coefficient $T(1,1)$. To select a suitable threshold $m$, we first 
estimated $T(1,1)$ for various thresholds. The results are shown on the left hand side of Figure 4 . The only small plateau in the plot can be found for threshold values between 60 and 70 . Therefore, we choose $m=60$ and obtain:

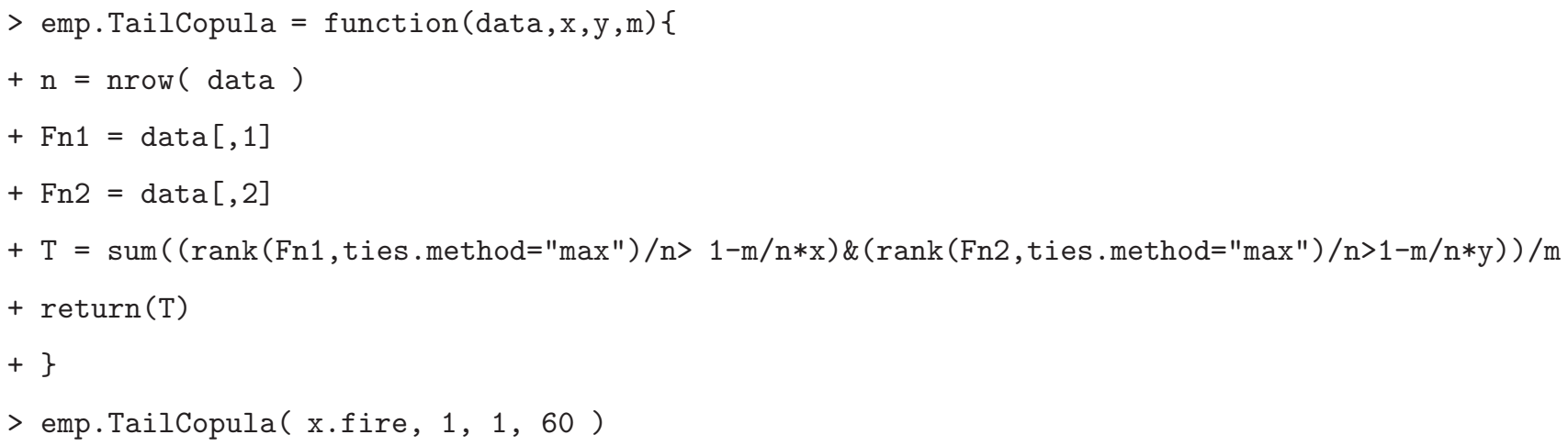

[1] 0.4166667

sample estimate: $\mathrm{T}(1,1 ; 60)=0.4166667$

This indicates the presence of upper tail dependence. To investigate this further, we estimate $T\left(x_{1}, x_{2}\right)$ at the points $\left(x_{1}, x_{2}\right)=(\sqrt{2} \cos (\varphi), \sqrt{2} \sin (\varphi)), \varphi \in\left(0, \frac{\pi}{2}\right)$, which contain all information about tail dependence in the upper right quadrant due to the homogeneity property (see e.g. Schmidt and Stadtmüller [56, Theorem 1]) of the tail copula. In Figure 4 we plot the smoothed tail copula estimator $\frac{n}{m} \hat{\mathbb{T}}_{n}(\sqrt{2} \cos (\varphi), \sqrt{2} \sin (\varphi))$ and the two jackknife confidence intervals

$$
I_{1-\alpha}^{T}\left(\sqrt{2} \cos (\varphi), \sqrt{2} \sin (\varphi) ; m, 1.5 m^{-1 / 3}\right), \quad \alpha \in\{0.05,0.1\}
$$

for $\varphi \in\left(0, \frac{\pi}{2}\right)$ and $m=60$. None of the given intervals covers zero confirming our assumption of tail dependence for the loss of building and the loss of contents.

\section{Conclusion}

Extreme value copulas and tail copulas are powerful dependence functions for the assessment of joint large risks, which create particularly dangerous situations in risk management. It is our firm believe that the statistical methods presented here will help to improve risk management in the financial industry. The statistical analyses we have presented in Sections 3.4 and 4.4 show that these methods work and are indeed applicable even with standardized software. It is also clear that further efforts are necessary to provide standardized methods and software for easy application in industry.

Since risk management problems in industry are often higher dimensional, it is important also to provide methods and software to assess risk in arbitrary portfolios. A straightforward dimension 

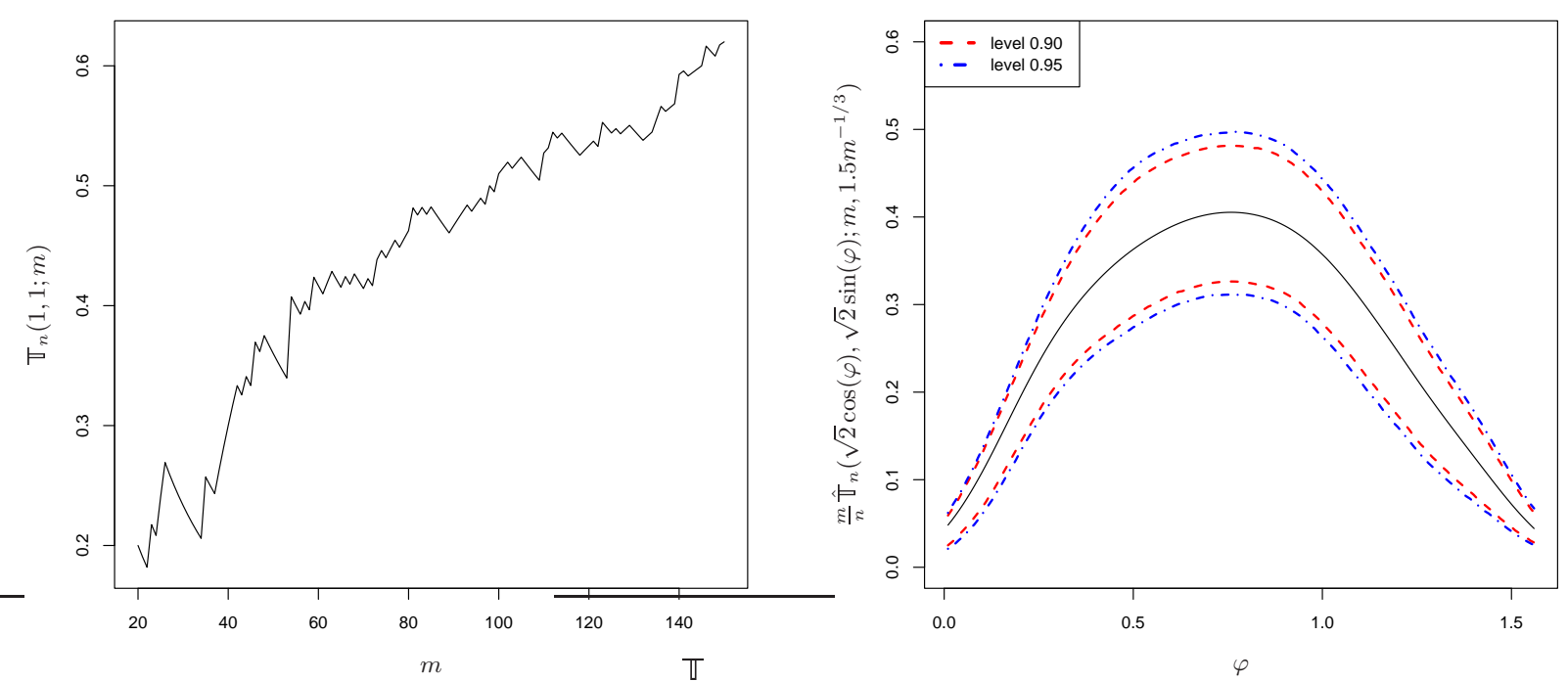

FiguRE 4. Left: Upper tail dependence coefficient estimate $\mathbb{T}_{n}(1,1 ; m)$ for various $m$. Right: Smoothed (solid) tail copula $\frac{n}{m} \hat{\mathbb{T}}_{n}\left(\sqrt{2} \cos (\varphi), \sqrt{2} \sin (\varphi) ; m, 1.5 m^{-1 / 3}\right)$ with $90 \%$ (dashed) and $95 \%$ (dotted-dashed) jackknife confidence interval for $\varphi \in\left(0, \frac{\pi}{2}\right)$ and $m=60$.

reduction method in the context of the present paper has been suggested in Haug, Klüppelberg and Kuhn [29], where also the necessary software is available.

\section{Acknowledgement}

Peng's research was supported by NSA grant H98230-10-1-0170 and NSF grant DMS-1005336.

\section{References}

[1] Beirlant, J., Goegebeur, Y., Segers, J., \& Teugels, J. (2004). Statistics of Extremes.John Wiley \& Sons.

[2] Ben Ghorbal, N., Genest, C., \& Nešlehová, J. (2009). On the Ghoudi, Khoudraji, and Rivest test for extreme-value dependence. The Canadian Journal of Statistics 37, 534-552.

[3] Berg, D. (2009). Copula goodness-of-fit testing: an overview and power comparison. The European Journal of Finance 15, 675-701.

[4] Bucher, A., \& Dette, H. (2010). Some comments on goodness-of-fit tests for the parametric form of the copula based on $L^{2}$-distances. Journal of Multivariate Analysis 101, 749-763. 
[5] Capéraà, P., Fougères, A.-L., \& Genest, C. (1997). A nonparametric estimation procedure for bivariate extreme value copulas. Biometrika $84,567-577$.

[6] Chan, N.H., Chen, J., Chen, X., Fan, Y., \& Peng, L. (2009). Statistical inference for multivariate residual copula of GARCH models. Statistica Sinica 19, 53-70.

[7] Chen, X., Fan, Y., \& Tsyrennikov, V. (2006). Efficient estimation of semiparametric multivariate copula models. Journal of the American Statistical Association 101, 1228-1240.

[8] Chen, J., Peng, L., \& Zhao, Y. (2009). Empirical likelihood based confidence intervals for copulas. Journal of Multivariate Analysis 100, 137-151.

[9] de Haan, L., \& Ferreira, A. (2006). Extreme Value Theory: An Introduction. Springer.

[10] de Haan, L., Neves, C., \& Peng, L. (2008). Parametric tail copula estimation and model testing. Journal of Multivariate Analysis 99, 1260-1275.

[11] Deheuvels, P. (1991). On the limiting behavior of the Pickands estimator for bivariate extremevalue distributions. Statistics \& Probability Letters 12, 429-439.

[12] Drees, H., \& Huang, X. (1998). Best attainable rates of convergence for estimates of the stable tail dependence functions. Journal of Multivariate Analysis 64, 25-47.

[13] Einmahl, J.H.J., de Haan, L., \& Li, D. (2006). Weighted approximations of tail copula processes with application to testing the multivariate extreme value distribution. The Annals of Statistics 34, 1987-2014.

[14] Einmahl, J.H.J., Krajina, A., \& Segers, J. (2008). A method of moments estimator of tail dependence. Bernoulli 14, 1003-1026.

[15] Embrechts, P. (2009). Copulas: a personal view. Journal of Risk and Insurance 76, 639-650.

[16] Embrechts, P., Klüppelberg, C., \& Mikosch, T. (1997). Modelling Extremal Events for Insurance and Finance. Springer, Berlin.

[17] Embrechts, P., McNeil, A., \& Straumann, D. (2002). Correlation and dependence in risk management: properties and pitfalls. In M.A.H Dempster (Ed.), Risk Management: Value at Risk and Beyond (pp. 176-223). Cambridge University Press.

[18] Esmaeili, H. and Klüppelberg, C. (2010). Parameter estimation of a bivariate compound Poisson process. Insurance: Mathematics and Economics 47, 224-233.

[19] Fermanian, J.D., Radulović, D., \& Wegkamp, M. (2004). Weak convergence of empirical copula processes. Bernoulli 10, 847-860.

[20] Gebhard, Ph., Müller, G., \& Böcker, K. (2010). Bayesian estimation of Lévy copulas for mul- 
tivariate operational risks. In : Böcker, K. (Ed.) Rethinking Risk Measurement and Reporting: Uncertainty, Bayesian Analysis and Expert Judgement, Vol. II. To appear in: Risk Books, London.

[21] Genest, C., Ghoudi, K., \& Rivest, L.-P. (1995). A semiparametric estimation procedure of dependence parameters in multivariate families of distributions. Biometrika 82, 534-552.

[22] Genest, C., Kojadinovic, I., Nešlehová, J., \& Yan, J. (2010). A goodness-of-fit test for extremevalue copulas. Bernoulli 17, 253-275.

[23] Genest, C., \& Remillard, B. (2004). Tests of independence and randomness based on the empirical copula process. Test 13, 335-369.

[24] Genest, C., Remillard, B., \& Beaudoin, D. (2009). Goodness-of-fit tests for copulas: a review and a power study. Insurance: Mathematics and Economics 44, 199-213.

[25] Genest, C., \& Segers, J. (2009). Rank-based inference for bivariate extreme-value copulas. The Annals of Statistics 37, 2990-3022.

[26] Ghoudi, K., Khoudraji, A., \& Rivest, L.-P. (1998). Propriétés statistiques des copules de valeurs extrêmes bidimensionnelles. The Canadian Journal of Statistics 26, 187-197.

[27] Gudendorf, G., \& Segers, J. (2010). Extreme-value copulas. In P. Jaworski, F. Durante, W.K. Härdle, \& T. Rychlik (Eds.), Proceedings of the Workshop on Copula Theory and its Applications (pp. 127-146). Springer.

[28] Hall, P., \& Tajvidi, N. (2000). Distribution and dependence-function estimation for bivariate extreme-value distributions. Bernoulli 6, 835-844.

[29] Haug, S., Klüppelberg, C., Kuhn, G. (2010) Copula structure analysis based on extreme dependence. Technical report.

[30] Huang, X. (1992). Statistics of Bivariate Extreme Values. Ph.D. Thesis, Tinbergen Institute Research Series.

[31] Jing, B.-Y., Yuan, J., \& Zhou, W. (2009). Jackknife empirical likelihood. Journal of the American Statistical Association 104, 1224-1232.

[32] Joe, H. (1997). Multivariate Models and Dependence Concepts. Chapman\& Hall, London.

[33] Joe, H., Li, H., \& Nikoloulopoulos, A.K. (2010). Tail dependence functions and vine copulas. Journal of Multivariate Analysis 101, 252-270.

[34] Kim, G., Silvapulle, M.J., \& Silvapulle, P. (2007). Comparison of semiparametric and parametric methods for estimating copulas. Computational Statistics $\&$ Data Analysis 51, 2836-2850.

[35] Klüppelberg, C., Kuhn, G., \& Peng, L. (2007). Estimating the tail dependence of an elliptical 
distribution. Bernoulli 13, 229-251.

[36] Klüppelberg, C., Kuhn, G., \& Peng, L. (2008). Semi-Parametric Models for the Multivariate Tail Dependence Function - the Asymptotically Dependent Case. Scandinavian Journal of Statistics $35,701-718$.

[37] Kolve, N., dos Anjos, U. and Mendes, B. (2006). Copulas: a review and recent developments. Stoch. Models 22, 617-660.

[38] Li, D., \& Peng, L. (2009). Goodness-of-fit test for tail copulas modeled by elliptical copulas. Statistics 83 Probability Letters 79, 1097-1104.

[39] Mesfioui, M., Quessy, J.F., \& Toupin, M.H. (2009). On a new goodness-of-fit process for families of copulas. The Canadian Journal of Statistcs 37, 80-101.

[40] Mikosch, T. (2006). Copulas: tales and facts. Extremes 9, 3-20.

[41] Molanes Lopez, E.M., Van Keilegom, I., \& Veraverbeke, N. (2009). Empirical likelihood for nonsmooth criterion functions. Scandinavian Journal of Statistics 36, 413-432.

[42] Omelka, M., Gijbels, I., \& Veraverbeke, N. (2009). Improved kernel estimation of copulas: weak convergence and goodness-of-fit testing. The Annals of Statistics 37, 3023-3058.

[43] Owen, A. (2001). Empirical Likelihood. Chapman \& Hall/CRC.

[44] Patton, A. (2009). Copula-based models for financial time series. In T.G. Anderson, R.A. Davis, J.P. Kreiss, \& T. Mikosch (Eds.), Handbook of Financial Time Series ( pp. 767-785). Springer: Berlin.

[45] Peng, L., \& Qi, Y. (2007). Partial derivatives and confidence intervals of bivariate tail dependence functions. Journal of Statistical Planning and Inference 137, 2089-2101.

[46] Peng, L., \& Qi, Y. (2008). Bootstrap Approximation of Tail Dependence Function. Journal of Multivariate Analysis 99, 1807-1824.

[47] Peng, L., \& Qi, Y. (2010). Smoothed jackknife empirical likelihood method for tail copulas. Test $19,514-536$.

[48] Peng, L., Qi, Y., \& Van Keilegom, I. (2011). Jackknife empirical likelihood method for copulas. Test, to appear.

[49] Peng, L., Qian, L., \& Yang, J. (2010). Weighted estimation of dependence function for an extremevalue distribution. Technical report.

[50] Pickands, J. (1981). Multivariate extreme value distributions. Bulletin of the International Statistical Institute 49, 859-878. 
[51] Qi, Y. (1997). Almost sure convergence of the stable tail empirical dependence function in multivariate extreme statistics. Acta Mathematicae Applicatae Sinica 13, 167-175.

[52] Qin, J., \& Lawless, J.F. (1994). Empirical likelihood and generalestimating equations. The Annals of Statistics 22, 300-325.

[53] R Development Core Team (2010). R: A language and environment for statistical computing. R Foundation for Statistical Computing, Vienna, Austria. ISBN 3-900051-07-0, URL http://www.Rproject.org.

[54] Rüschendorf, L. (1976). Asymptotic distributions of multivariate rank order statistics. The Annals of Statistics 4, 912-923.

[55] Rytgaard, M. (1996). Simulation experiments on the mean residual life function $m(x)$. In: Proceedings of the XXVII Astin Colloquium (pp. 59-81). Copenhagen, Denmark.

[56] Schmidt, R., \& Stadtmüller, U. (2006). Nonparametric estimation of tail dependence. Scandinavian Journal of Statistics 33, 307-335.

[57] Tawn, J. (1988). Bivariate extreme value theory: models and estimation. Biometrika 75, 397-415.

[58] van der Vaart, A.W. (1998). Asymptotic Statistics. Cambridge University Press.

[59] Zhang, D., Wells, M.T., \& Peng, L. (2008). Nonparametric estimation of the dependence function for a multivariate extreme value distribution. Journal of Multivariate Analysis 99, 577 - 588 . 